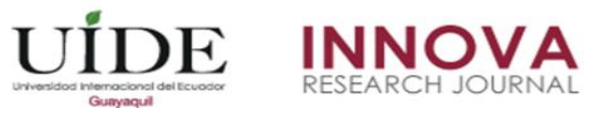

INNOVA Research Journal, ISSN 2477-9024

(Enero, 2018). Vol. 3, No.1 pp. 30-41

DOI: https://doi.org/10.33890/innova.v3.n1.2018.336

URL: http://revistas.uide.edu.ec/index.php/innova/index

Correo: innova@uide.edu.ec

\title{
Las competencias argumentativas en la formación universitaria
}

\section{The argumentative competencies in the university education}

Raisa Araminta Torres Ruíz

María Gabriela Ochoa Villa

Universidad Iberoamericana del Ecuador UNIB.E, Ecuador

Dargenis La Rosa Torres

Universidad Politécnica Salesiana, Ecuador

Ana Belén García Berbén

Universidad de Granada, España

Autor para correspondencia: rtorres@unibe.edu.ec,mochoa@unibe.edu.ec,dla@ups.edu.ec, berben@ugr.edu.es

Fecha de recepción: 26 de Julio 2017 - Fecha de aceptación: 15 de Enero de 2018

Resumen: El tema de la formación por competencias en la Educación Superior ecuatoriana ha ido tomando cada vez más relevancia en las aulas. Sin embargo el trabajo de las competencias argumentativas aún sigue estando relegado. La presente investigación se realizó con el objetivo de analizar las diferentes aristas del concepto y demostrar que la competencia argumentativa, vista como una habilidad social resulta de gran importancia en el desempeño laboral, social y personal y como elemento fundamental en el pensamiento conceptual de nuestros estudiantes universitarios y futuros profesionales. Para lograr una visión holística de este concepto, se decantan las debilidades en su consecución dentro de la enseñanza universitaria actual, retomando los primeros estudios al respecto y reconociendo las primeras investigaciones, postulados y precursores. Así mismo la investigación incluye una revisión de las investigaciones y definiciones prácticas que actualmente se manejan sobre el tema.

Palabras clave: competencias argumentativas; educación superior; conocimiento de la teoría de la argumentación; implementación de la teoría de la argumentación

Abstract: The subject of competency-based training in higher education in Ecuador has been taking on more and more relevance in the classroom. However, the work of the argumentative powers is still relegated. This research was conducted with the objective of analyzing the different edges of the concept and demonstrate that argumentative competition, seen as a social skill is of great importance in the work, social and personal performance and as a fundamental element in the conceptual thinking of our university students and future professionals. To achieve a holistic view of this concept, the weaknesses in its attainment are decanted in the current university education, taking up the first studies in this respect and recognizing the first investigations, postulates and precursors. The research also includes a review of the research and practical definitions that are currently handled on the subject.

Key words: argumentative competences; higher education; knowledge of the theory of argumentation; implementation of the theory of argumentation 


\section{Introducción}

El tema de competencias en el ámbito de la educación se encuentran desde mediados de la década de los noventa en expresiones tales como formación por competencias, planes de estudio basados en el enfoque por competencias, propuestas educativas por competencias, entre otras, presentándose en sus diversas formas, como una opción alternativa para mejorar los procesos de formación académica tanto en el nivel de educación básica como en la formación del técnico medio y la formación de profesionales con estudios de educación superior (Ríos, 2013).

Sin embargo, cuanto a la argumentación los estudiantes exhiben dificultades en esta área del pensamiento dado que no ha tenido la suficiente atención, y se hacen patentes en las evaluaciones, con lo que vienen preocupando a la comunidad educativa desde hace tiempo. En principio, es posible considerar que a partir de la transformación de las comunicaciones, el uso de la cultura letrada que los jóvenes realizan en la actualidad se diferencia notablemente de las anteriores generaciones. Esto parece estar relacionado con los cambios en el aprendizaje y entrenamiento de la lectoescritura. (Araminta, Carmona, \& La Rosa, 2017).

\section{Desarrollo}

En el desarrollo realizaremos un análisis del concepto de competencia argumentativa desde diferentes puntos de vista y su papel en el adecuado desempeño laboral, social y personal como componente del pensamiento conceptual.

\section{Aproximación conceptual al tema de las competencias argumentativas.}

La competencia argumentativa es concebida como una habilidad a adquirir, tiene una importancia crucial como método de desarrollo del pensamiento en el mundo académico a lo que se puede sumar su implicación social en todo proceso de enseñanza-aprendizaje (Crowell \& Kuhn, 2014). El desarrollo y estimulación de las competencias en la argumentación señalan una dirección hacia un aula de clase más humanizada, que potencia la actitud participativa del alumnado, guiándolo hacia un aprendizaje propositivo y exploratorio a partir del nuevo tipo de relaciones, que son establecidas para facilitar el desarrollo de la inteligencia del sujeto a través de la estimulación de los procesos mentales superiores (Arenas, 2014).

Es una habilidad compleja que se enmarca dentro de los procesos superiores del pensamiento e implica no solo conservar la posición o idea concreta, sino saber defenderla, disentir de forma respetuosa y razonada de las opiniones que expresan otras personas, analizar la información recibida con criterios flexibles y respaldados y poder enjuiciarla posicionándose de forma motivada ante los desacuerdos, para ello resulta necesario que se comprendan los disímiles criterios y aristas implicadas en los conflictos, encontrar y proponer alternativas para las explicaciones y si es necesario cambiar la perspectiva propia o ayudar al cambio de visión en los demás, por lo que resulta básica para una adecuada convivencia en sociedad y para ello debe constituir un objetivo didáctico esencial del ámbito educativo (García A. , 2015 ).

A juicio de la autora, las dificultades que exhiben los estudiantes de nivel universitario en esta área del pensamiento, se hacen patentes en las evaluaciones y vienen preocupando a la comunidad educativa desde hace tiempo. En principio, es posible considerar que a partir de la 
transformación de las comunicaciones, el uso de la cultura letrada que los jóvenes realizan en la actualidad se diferencia notablemente de las anteriores generaciones y parece estar relacionado con los cambios en el aprendizaje y el entrenamiento de la lectoescritura.

Lo anterior se hace manifiesto en la escritura epistolar, los trabajos monográficos y de investigación escolar que tradicionalmente se utilizaron como adiestramiento en las habilidades de escritura y que han dado paso a la utilización de todo tipo de escritura abreviada. Se observan textos escolares donde predomina lo hipertextual, mensajes de textos que por su síntesis se podrían considerar como pseudo-encriptados, que dan cuenta de un mayor consumo de textos publicitarios y menos consumo de literatura (Garay, 2014).

De acuerdo a las observaciones de la autora, muchos alumnos tienen dificultades de comprensión lectora, lo que de alguna manera condiciona la competencia de producir textos y mucho más de argumentar. Estas dificultades pueden llegar a obstaculizar o condicionar la inserción laboral de los jóvenes. En todo caso, uno de los factores claves que deben tenerse en cuenta, a la hora de abordar esta problemática, es la importancia de la lectura y de la práctica argumentativa para poder mejorar las competencias inherentes a poder sostener un argumento, afrontar una discusión o un diálogo desde diferentes perspectivas y tomando en cuenta las principales dimensiones de la práctica argumentativa (meta cognitiva, epistemológica y social).

El presente estudio reviste una importancia práctica, pues en distintos campos de conocimiento también se vienen dando, a juicio de la autora, nuevos desarrollos teóricos fundamentados por los avances científicos y prácticas comunicativas, que demandan particularmente habilidades argumentativas por parte de los futuros profesionales.

Desde un enfoque pedagógico y lingüístico, teniendo en cuenta el contacto y la comunicación que debe establecerse entre los docentes y la sociedad, resulta indispensable que los estudiantes mejoren sus competencias y habilidades argumentativas, así como su disposición a argumentar y todo lo que concierne a la ejercitación de la lectura y la elaboración de escritos herramientas fundamentales en la era de la globalización-, para propiciar una buena inserción laboral (Garcia, Condat, Occelli, \& Valeiras, 2016).

De acuerdo con Means y Voss (1996), la capacidad de argumentar actúa como una habilidad intelectual de orden superior, se considera un proceso particular del pensamiento crítico extremadamente difícil de enseñar y está altamente implicada para alcanzar el éxito en la participación social. (Romero, 2012).

Para poder diseñar y ejecutar algún programa pedagógicos orientado al desarrollo de la argumentación es necesario, en primera instancia, diagnosticar el nivel alcanzado en el desarrollo de la argumentación en los estudiantes y planificar los métodos más adecuados para el desarrollo de estas habilidades, así como sus disposiciones hacia el trabajo intelectual que demanda argumentar, lo que se intentará hacer en la presente investigación. Lamentablemente, son escasos los estudios que se han realizado en esta área del conocimiento. La literatura revisada sobre el desarrollo de la argumentación no es clara acerca de la estandarización, evolución y resultados a esperar de estas capacidades en cada nivel educativo por los estudiantes. 
Si bien se destaca que la argumentación es una habilidad de importancia crítica en la vida social del siglo XXI y en la cual los educadores han comenzado a focalizar su atención, poco se sabe sobre cómo los estudiantes desarrollan sus competencias argumentativas y por consiguiente, cómo es posible enseñarlas o estimularlas. De todos modos, el desarrollo de las competencias argumentativas se perfila como una cuestión crítica en el campo de la enseñanza universitaria, tanto en el plano teórico como en el práctico (García A. , 2015 ).

En Latinoamérica la investigación sobre la actividad argumentativa se han estado centrando en el problema de la complejidad argumentativa, se reconocen investigaciones para establecer el esquema argumentativo más recurrente empleado en la producción de textos escritos por un grupo de estudiantes de tercer año de enseñanza media en Chile, sobre la base de que el estudio del uso de esquemas argumentativos puede proyectar el formas de razonamiento característicos del fondo de los hablantes; así como acerca de los aspectos dialécticos y retóricos más destacados en los diálogos argumentativos entre los estudiantes de dos establecimientos educacionales. Son varios los estudios además que revelan bajos niveles de "argumentatividad". (Aguiar, 2016; Araminta, Carmona, \& La Rosa, 2017; López \& Padilla, 2011).

\section{Modelos de argumentación de Toulmin y Perelman.}

Lo que hoy conocemos como teoría de la argumentación tiene una historia reciente. En la segunda mitad del pasado siglo autores como Perelman y Toulmin atrajeron la curiosidad acerca de la naturaleza de la argumentación dentro del lenguaje natural y la carencia en cuanto a propuestas de algún modelo normativo adecuado para ella. Sus teorías dieron apertura a estudios que se han distinguido como un devenir de la lógica, la oratoria y la dialéctica dentro de las conceptualizaciones sobre la teoría de la argumentación (Forsberg, 2015).

En 1958 aparece en Inglaterra el libro The uses of argument de Stephen Toulmin, su propósito fue dar a conocer un cuestionamiento epistemológico que se propuso criticar el modo tradicional de entender la producción del conocimiento y el hábito en la ciencia de suponer como fundamento del razonamiento, el proceso silogístico aristotélico incluso para la cotidianidad de la vida (Murillo, 2013).

A partir del tercer capítulo de su libro, se encuentra su proposición bajo la denominación de: lógica factual. El interés de la propuesta estaba en examinar la forma en que el razonamiento se desarrolla en un contexto específico de análisis. El libro en específico, está centrado en el proceso de la jurisprudencia y su ejercicio, realiza un análisis acerca del peso de las leyes en relación a la solución de casos y los criterios de aplicación de la fuerza y el criterio, que pasan a ser dos términos claves para alcanzar una representación indiscutible del planteamiento de los argumentos (Toulmin, 1958).

Esta propuesta fue establecida por Toulmin montando un modelo con seis entradas, que según Marmolejo (2016) supera la lógica aristotélica que se concentraba primordialmente en los aspectos premisa mayor, premisa menor y conclusión.

El modelo que fundamenta su método para confeccionar y analizar los argumentos, presenta las entradas siguientes: 1). Apoyo: define los contenidos que dan fundamento a las 
garantías y que provienen de investigaciones, libros, códigos, supuestos sociales que permiten garantizar una garantía, 2). Garantía: es el principio general, la norma tácita, el supuesto o enunciado general que reviste naturaleza formal y que permite el paso la transición de datos a conclusiones, 3).Datos: conforman el cuerpo empírico de orden factual que permite fundamentar que surja la pretensión o conclusión, 4).Conclusión: constituye la pretensión en que se basa la demanda o alegato y tiene como propósito, argumentar la acción o perspectiva, 5) Cualificador modal: se trata de una construcción lingüística que apoya el cambio de la fuerza pragmática para establecer una pretensión y 6) Condición de refutación: son las excepciones que pueden debilitar el establecimiento de garantías, son parte de la información contenida en la fundamentación del apoyo y pueden socavar la fuerza conclusiva de la conclusión.

El modelo es perfectamente aplicable en la enseñanza y práctica de esta habilidad por su progresivo manejo de las categorías argumentativas y se interpreta de la forma siguiente: "dada la experiencia general sobre el campo en cuestión (apoyo) en conformidad con las reglas o principios resultantes de tal experiencia (garantía), con la utilización de los siguientes hechos específicos (datos) y de una forma cualificada (cualificador modal), se consiente en la siguiente conclusión (conclusión o pretensión), a menos que se establezca una específica condición de refutación (excepciones).

Como se infiere del nombre "lógica factual" esta denominación se estable porque en el modelo se establece una relación dinámica con las situaciones y acontecimientos, que se visualizan tanto a través de los "apoyos", los "datos" y "excepciones", como de la "conclusión" y los "cualificadores modales" que se entrelazan en las estrategias que usan las personas para establecerse en debates y controversias.

El renacimiento de la retórica se produce a finales de los cincuenta del pasado siglo XX, fundamentalmente los estudios de la Nueva Retórica de Chaïm Perelman y Lucie OlbrechtsTytec, Perelman en estos, se aprecia el rechazo a la condena del irracionalismo en las cuestiones relacionadas con los valores, propugnado por la filosofía del positivismo lógico (Sanchez, 2015) y creará las bases de una lógica interdisciplinar, aglutinando diversas aportaciones entre las cuales encontramos, los estudios de psicología experimental de las audiencias y de la sociología del conocimiento. La base de sus propuestas se encuentra en los siguientes principios de la dialéctica de Gonseth:

1. Principio de integridad: el saber es íntegramente interdependiente.

2. Principio de dualismo: no existe dicotomía entre los métodos racional y empírico; ambos deben reconciliarse.

3. Principio de revisión: cualquier afirmación o principio estará sujeto a nuevos argumentos, que podrán anularlo, debilitarlo o reforzarlo.

4. Principio de responsabilidad: la personalidad del investigador estará comprometida en sus afirmaciones y teorías, por lo que las justificaciones serán racionales y estar sujetas a cambios.

Naturalmente, el objeto de esta teoría es el estudio de las técnicas de discurso que pueden ser capaces de estimular o aumentar la adhesión de las persona a los juicios presentados para su aceptación (Perelman, 1989). 
Desde los desarrollos conceptuales de estos precursores se ha intensificado la atención sobre el tema de la argumentación y su carácter de habilidad o competencia al servicio del aprendizaje significativo y autónomo, preocupación de carácter internacional que ha incentivado un movimiento por a favor de las reformas en los sistemas de educación, buscando nuevas formas de estructurar los currículos y disposiciones para entender los procesos de enseñanzaaprendizaje.

\section{Aspectos básicos de la habilidad argumentativa.}

En un libro publicado acerca de los estudios realizados por Van Eemeren y Grootendorst (1992), quienes reformularon la teoría del análisis crítico, se asegura que de principio, la noción de argumentación que postulan requiere de una controversia y de la discusión crítica de puntos de vista opuestos, constituyendo estos elementos centrales del proceso argumentativo (Carretero, 2015).

Para estos autores, la argumentación surge cuando dos o más personas sostienen puntos de vista divergentes, que de alguna manera, intentan llegar a un acuerdo y como necesidades de segundo y tercer orden plantean la condición emocional de los interlocutores y las condiciones en que la discusión se desarrolla. Considerando estos tres elementos un marco bastante completo para el análisis y comprensión del proceso argumentativo.

De acuerdo con (Leitão, 2006), en un libro publicado sobre Psicología Cognitiva: cultura, desenvolvimiento y aprendizaje; la argumentación se presenta no únicamente cuando se intentan resolver posiciones opuestas, que se encuentran formuladas de manera explícita, de igual forma se pueden presentar implícitamente ante una justificación o posición alternativa. La justificación surge cuando hay elementos que evidencian debilidad en las opiniones, estas son relativas a la firmeza de las opiniones alternativas. Desde la perspectiva presentada, la justificación sería el punto de cambio que transforma un discurso en argumentación ( Larraín, Freire, \& Olivos, 2014).

En todo caso, se parte de una concepción de la argumentación que tiene en cuenta su carácter socio-discursivo, dialógico y dialéctico, que promueve la negociación de puntos de vista a partir de diferencias de opinión. Estas diferencias provienen incluso de diversas fuentes como la prensa escrita o hablada y la literatura, entre otras formas de difusión de las ideas y no únicamente de los participantes presentes en un intercambio comunicacional, donde la argumentación es producida de forma inmediata, en este cuadro puede sumarse el llamado discurso interior, donde las argumentaciones se plantearían al sí mismo (Van Eemeren, Grootendorst , \& Snoeck , 1996).

Según Leitão (2006) la argumentación vista como unidad constituye el ensamblaje entre la posición justificada y una idea contrapuesta justificada (que sería el contra-argumento) junto a la respuesta o tercera posición. Los mecanismos de interpretación entre los significantes y significados para cada persona favorece la evaluación de las posiciones cognitivas del hablante y determina un recurso medular en la construcción y de-construcción del conocimiento. Varios autores coinciden que el contra-argumento es un mecanismo semiótico importante durante la 
conformación conocimiento, al forzar los fundamentos de cada idea en cuestionamiento (Toulmin, 1958; Perelman \& Olbrechts-Tyteca, 1989).

En consecuencia la intervención durante la argumentación de posiciones opuestas formuladas explícitamente junto a las justificaciones será un proceso más enriquecedor de desarrollo cognitivo que sólo con justificación. En una mirada más profunda de este proceso, inicialmente se partirá del momento en que no hay alguno de los mecanismos semióticos de los ya descritos por Leitão (2000), aun el discurso no será argumentativo, será con la aparición de la demanda o justificación que surgen al menos dos posiciones contrapuestas: "la que se sostiene" a ser fundamentada y por otra parte la que compite con la primera.

Las evidencias anteriores propuestas por este autor, llevan a la idea que es la justificación, la que da apertura al discurso, aportando posiciones alternativas aun cuando no estén formuladas de forma explícita, es cuando se suma el contra-argumento, en un segundo momento que se presenta la oposición explícita, ya dirigida hacia el argumento "posición más justificación" planteado con anterioridad. El planteamiento, según (Leitão, 2006), impele al sujeto a considerar y revisar las posiciones sostenidas hasta ese momento y los fundamentos de las mismas.

Finalmente se presenta la respuesta, como el tercer movimiento planteado por el autor, con este mecanismo semiótico se da paso a la evaluación de las posiciones ya esgrimidas y la conformación de una idea propia.

En otro orden, los estudios sobre el razonamiento infantil plantean que las estructuras argumentativas se dan en una forma simple, es decir conclusión y una razón; en modo fortalecido, cuando se presentan las anteriores más uno o más modalizadores y la tercerea forma sería la elaborada, que es cuando aparecen el argumento y su contra-argumento. La presencia de la estructura fortalecida amplía en su frecuencia de acuerdo al nivel educativo de la persona, sin embargo en la estructura elaborada no se observa tal incremento. Por ejemplo, los niños de grados inferiores con alto nivel cognitivo pueden tener mejores desempeños argumentativos que otros de grados superiores que presentan bajos niveles en el desarrollo cognitivo (Means \& Voss, 1996).

Esta situación sugiere dos cuestiones importantes. De un lado, las investigaciones de estos autores muestran q la habilidad de contra-argumentación aún no se desarrollan totalmente en la mayoría de los chicos de $11^{\circ}$ grado, o según Glassner y Schwarz, (2005) la habilidad de "anti-logos" estaría en pleno desarrollo en este período, cuando se dan condiciones apropiadas. De lo que se concluye que las habilidades para la argumentación, no sólo avanzan con la edad, se desarrollan más bien gracias a la posibilidad de exponerse de forma sistemática a experiencias participativas que involucren el debate o instancias que demandan de estas habilidades.

\section{Dimensiones en el desarrollo de la argumentación.}

De acuerdo a Larraín et al. (2014) el debate en torno a la adquisición y puesta en juego de habilidades argumentativas, debería sustentarse al considerar y distinguir al menos tres dimensiones que resultan imprescindibles, la estructura argumentativa, la dimensión comprensiva-productiva y la dimensión social: 
1. Estructura argumentativa: esta se va fortaleciendo a lo largo del desarrollo ontogenético y se puede observar su avance desde una estructura justificativa a una ya con desarrollo dialéctico, que sostiene la capacidad para mantener los puntos de vista, elaboración y justificación de posturas o fundamentos que la contradicen. El resultado de las investigaciones en torno a este problema a detallado que los niños de temprana edad ya son capaces de plantear sus posiciones y proveer más razones según avanza el dialogo para sustentar las mismas y elaborar contra-argumentos para defenderlas. Es este atributo dialéctico, según la literatura científica disponible acerca de la producción argumentativa, el más difícil de alcanzar (Glassner \& Schwarz, 2005).

2. Dimensión comprensiva-productiva: en una revisión metódica de las indagaciones al respecto, destaca que tempranamente los niños serán capaces de argumentar y permite dar cuenta de que se centran más bien en capacidades comprensivas: serán capaces de saber e identificar la estructura del argumento a temprana edad. No obstante, con respecto a la habilidad productiva, se observa que los pequeños son capaces de producir mejor las propias justificaciones para mantener sus puntos de vista (Leitão, 2006). En este sentido los niños/as de corta edad pueden ofrecer durante una discusión más razones que apoyan sus propios puntos de vista, a los que pueden aportar para apoyar los puntos de sus oponentes (Stein \& Albro, 2001).

Sobre este particular se suscitan contradicciones en la literatura pues algunos autores consideran que los niños no comprenden un contra-argumento a una postura ajena o propia desde muy temprano, pues esto estaría acompañado de un desarrollo progresivo y paulatino de habilidades de comprensión, que implica entender las debilidades de un argumento.

De hecho, otros autores confirman esta deducción y terminan concluyendo que a los cinco años los niños de ambos sexos demuestran dificultades para identificar las debilidades en los argumentos opuestos ( Larraín, Freire, \& Olivos, 2014). En efecto, se ha demostrado que es en la edad escolar donde la posibilidad de comprender la debilidad de un argumento opuesto va apareciendo. De todos modos, parece no haber grandes diferencias al respecto entre los niños mayores y adultos, resulta interesante que estos últimos no necesariamente demuestran un aumento significativo para el reconocimiento sus propias debilidades con respecto a las fortalezas del contrario cuando ejercitan sus habilidades argumentativas (Migdalek, Santibáñez, \& Rosemberg, 2014).

En términos temporales, Glassner y Schwarz (2005) postulan será al comienzo de la adolescencia, cuando la habilidad para contra-argumentar estarían en máximo desarrollo, pero no sería hasta la adultez que estas alcanzarán su completo desarrollo. Se puede adelantar la hipótesis de que las habilidades comprensivas son las que apoyan el desarrollo de la capacidad productiva. De acuerdo a las reflexiones precedentes, a nivel taxonómico es crucial diferenciar la comprensión versus elaboración, que se involucran en los diferentes aspectos de la habilidad para argumentar (justificación y oposición).

1. Dimensión social: es básico distinguir de forma adecuada la importancia del contexto social donde se ejercitan las habilidades de argumentación. La producción argumental se comparte con mayor responsabilidad en los contextos interpersonales, donde se demuestra la capacidad cognitiva, ello fundamenta que los estudiantes demuestren sus 
habilidades argumentativas mejor que cuando sólo un interlocutor interviene en las propuestas y se opone a las posiciones planteadas. Es en el diálogo, donde se co-producen recursos argumentativos con mayor calidad, entablándose la discusión según roles alternos, que contribuyen al desarrollo de la habilidad al intercambiar mediante argumentos sucesivos y contra-argumentos en virtud de las habilidades de cada parte (Ríos, 2013).

Desde una perspectiva contraria, en la construcción individual de un texto escrito con coherencia, la información debe estructurarse mediante una reflexión que hace la persona internamente, para lo cual debe generar y coordinar por sí misma las afirmaciones y contraargumentos que sustentan y se oponen a la declaración que se pretende exponer. En materia de calidad cognoscitiva, sin dudas esta es una tarea de más alto rango y demanda de mayores recursos para la estructuración de ambos puntos de vista (Piolat, Roussey, \& Gombert, 1999).

Desde la perspectiva de estos autores, es lógico concluir que el individuo muestra mejor desempeño cuando puede argumentar con los otros que cuando debe encargarse de la propuesta y oponencia del mismo enfoque. No es similar, desde el punto de vista del desarrollo, contraargumentar las ideas ajenas que las propias. Lo último requiere la experiencia de ejercer la contra-argumentación y la "internalización" de la estructura característica de las prácticas argumentativas, de modo que se puedan realizar sin una confrontación real. Se requiere de capacidades para el descentramiento, que implica llevar de forma conjunta las perspectivas propias y las ajenas.

Como síntesis de estos aportes la evidencia indica, que no sólo es una ganancia que se da sólo al llegar a la adultez, la posibilidad de elaborar los contra-argumentos para los demás, sino que identificar las propias debilidades, reconocer y elaborar contra-argumentos dirigidos hacia la propia posición y responderlos, son ganancias que se dan en etapas posteriores del desarrollo y únicamente alcanzan un alto nivel en presencia de una situación social favorecedora del desarrollo argumentativo.

Para comprender el desarrollo de la argumentación con el propósito de aprovecharlo desde una perspectiva pedagógica, es importante identificar el grado en que las habilidades argumentativas se desarrollan de acuerdo a las tres dimensiones ya descritas. Se debe entender de acuerdo a las posibilidades de desarrollo de la capacidad argumentativa cuando es que los niños presentan dificultades en elaborar contra-argumentos porque sin dudas es más fácil para ellos identificarlos. De igual manera son diferentes las posibilidades para identificar debilidades y fortalezas en los oponentes que para realizar el mismo ejercicio pero con respecto a las debilidades de las propias posturas (García A. , 2015 ).

En cuanto al desarrollo de la argumentación en la escritura, si bien existen importantes elementos que diferencian a las habilidades de argumentación escrita de las orales, la literatura acerca del tema da cuenta de que ambos patrones de construcción de la argumentación discurren por momentos similares en su desarrollo. Algunos autores sugieren que la argumentación escrita muestra un desarrollo que va desde niveles pre-argumentativos (es decir no hay fundamentación para sustentar la posición), luego un nivel argumentativo mínimo donde se sustenta la posición 
con al menos una razón y en su máximo desarrollo argumentativo ya más elaborado, se presentan múltiples elementos que se articulan como sustento o razones para demostrar las posiciones (Ramos, Sánchez, \& Huapaya, 2014).

Para entender las dificultades que involucra la argumentación escrita, parece ser relevante el nivel de autonomía que requiere a lo cual se suman las dificultades que implica el dominio del lenguaje escrito durante el curso por los diferentes niveles educativos y de forma particular la tardía adquisición de elementos para ejercer la contra-argumentación (Larraín, Freire, \& Olivos, 2014).

La argumentación en general puede verse favorecida por condiciones contextuales tales como la cualidad polémica del tema a debatir, la familiarización o experiencia previa de la audiencia, el objetivo del planteamiento, las características de las instrucciones, conocimientos, las creencias y los compromisos que la persona posea con relación al tema, las relaciones de simetría, entre otros aspectos y variables a considerar (Kuhn \& Udell, 2003).

En todo caso, para la autora es claro que la educación superior y los docentes encargados de la formación de estudiantes universitarios, deben tener presente que el proceso de adquisición de esta habilidad será progresivo, incluso a este nivel de enseñanza y logrará exteriorizar los procesos de pensamiento y del razonamiento, contrastar las hipótesis y juicios emitidos con sus pares y profesores, en la medida que sea objeto de entrenamiento durante el proceso de construcción y regulación del proceso de formación y apropiación del conocimiento.

La argumentación tiene relación con los objetivos que persiguen la formación de individuos responsables, que participen en decisiones sociales a través del pensamiento crítico (Jiménez, 2012). Propiciar la argumentación en las aulas para contribuir a un mejor aprendizaje en los estudiantes sería una buena estrategia a utilizar por parte de los profesores, que no puede quedarse solo en un discurso de buenas intenciones plasmado en los documentos oficiales de las instituciones educativas (Cruz, 2015).

La competencia argumentativa es la habilidad del razonamiento que permite explicar y entender el orden de las partes de un proceso, así como relación que existe entre estas partes para llegar a una conclusión. De tal manera que al argumentar se presenta una explicación acerca del porqué de las cosas, justificando a través de razones, para llegar a criterios propios (Crowell \& Kuhn, 2014; Cruz, 2015).

\section{Conclusiones}

Después de analizar las diferentes aristas del concepto y perspectivas de varios autores, podemos concluir que la competencia argumentativa, vista como una habilidad social, resulta de gran importancia en el desempeño laboral, social y personal. Constituye un elemento fundamental en el pensamiento conceptual de nuestros estudiantes universitarios y futuros profesionales. Por lo que debe transversalizar la actividad académica y curricular en nuestras aulas, logrando elevar la calidad en la formación profesional.

\section{Bibliografía}


Aguiar, M. d. (2016). Competencias argumentativas en estudiantes de educación superior y su relación con las creencias epistemológicas. Granada: Tesis. Universidad de Granada. Departamento de Métodos de Investigación y Diagnóstico en Educación.

Araminta, R., Carmona, M., \& La Rosa, D. (2017). La formación de las competencias argumentativas. Un reto dentro de la enseñanza superior. Revista Publicando, 3(9), 299-314.

Arenas, M. d. (2014). El concepto de competencias visto desde las tesis de grado de la Maestría en Educación y Desarrollo Humano de los años 1997 a 1er semestre de 2006 en la Línea de Desarrollo Cognitivo y Emotivo. Umanizales, Disponible en: http://ridum.umanizales.edu.co:8080/xmlui/handle/6789/1195.

Carretero, F. L. (2015). Argumentación y pragma-dialéctica: Estudios en honor a Frans van Eemeren. Guadalajara. Mejico: Editorial Universitaria. Disponible en: https://books.google.com.ec/books?id=loQHCwAAQBAJ\&pg=PT142\&lpg=PT142\&dq=Van + Eemeren+y+Grootendorst+(1992)\&s.

Crowell, A., \& Kuhn, D. (2014). Developing Dialogic Argumentation Skills: A 3-year Intervention Study. Journal of Cognition and Development, 15:2, 363-381. Disponible en: http://www.tandfonline.com/doi/abs/10.1080/15248372.2012.725187.

Cruz, M. d. (2015). Competencias argumentativas en estudiantes de la educación Superior y su relación con las creencias epistemológicas. Granada: Tesis publicada. Universidad de Granada.

Forsberg, L. (2015). Teoría de la Argumentación. A 50 años de Toulmin y Perelman. Disponible en: https://unlzsocialesresumenes.files.wordpress.com/.../teorc3adas-de-la-argumentacic3b...

Garay, G. O. (2014). Complejidad y deformación informativa en el lenguaje multimedia y la comunicación interactiva. Ambitos: Revista internacional de comunicación, ISSN-e 11391979, $\quad N^{o}$. 24, 81-90. Disponible en: https://dialnet.unirioja.es/servlet/articulo?codigo $=4678889$.

García, A. (2015 ). Importancia de la competencia argumentativa en el ámbito educativo: una propuesta para su enseñanza aImportancia de la competencia argumentativa en el ámbito educativo: una propuesta para su enseñanza a través del role playing online. Revista de Educación a Distancia. Número 45. 15-Mar, Disponible en: http://www.um.es/ead/red/45.

Garcia, L., Condat, M. E., Occelli, M., \& Valeiras, N. (2016). La dimensión argumentativa y tecnológica en la formación de docentes de ciencias. Ciênc. educ. (Bauru) vol.22 no.4 Bauru oct./dic, Disponible en: http://www.scielo.br/scielo.php?script=sci_arttext\&pid=S1516$73132016000400895 \& \operatorname{lng}=$ es\&nrm=iso\&tlng=es.

Jiménez, I. V. (2012). La perspectiva de género desde el estudiantado de práctica supervisada de la carrera de orientación en la UNA. Actualidades investigativas en Educación, Volumen 12, Número 2, Disponible en: Disponible en: http://www.redalyc.o. 
Larraín, A., Freire, P., \& Olivos, T. (2014). Habilidades de argumentación escrita: Una propuesta de medición para estudiantes de quinto básico. Disponible en http://www.psicoperspectivas.cl/index.php/psicoperspectivas/article/viewFile/287/316.

Kuhn, \& Udell. (2003). The development of argument skills. In Child Development, 74,, 1245-1260.

Leitão, S. (2006). Argumentación infantil: conductas opositivas y anticipación de opocición. En Psicologia Cognitiva: Cultura, Desenvolvimento E Aprendizagem. Cap 9. Pernambuco: Editora Universitaria UFPE. Disponible https://books.google.com.ec/books?id=P1Zjw4xODjsC\&pg=PA256\&dq=Leitão+(2000),+la+ argumentación\&h.

López, E., \& Padilla, C. (2011). Grados de complejidad argumentativas en escritos de estudiantes universitarios de Humanidades. PRAXIS.Revista de Psicología, Año 13, No. 20, 61-89.

Marmolejo, a. R. (2016). Modelo De Argumentación De Toulmin. Grado Cero Prensa, Disponible en: https://gradoceroprensa.wordpress.com/2016/06/02/modelo-de-argumentacion-de-toulmin/.

Means, M., \& Voss, J. (1996). Who reasons well? Two studies of informal reasoning among children of different grade, ability, and knowledge levels. In Cognition and Instruction, 14, 139-178.

Murillo, O. M. (2013). El Modelo Argumentativo De S. Toulmín Y Su Utilidad. Corporación Universitaria Rafael Núñez, Disponible en: http://www.curn.edu.co/lineas/escritura/1237modeloargumentativo.html.

Perelman, C. (1989). Tratado de la Argumentación. Madrid: Gredos. Disponible en: http://www.fis.cinvestav.mx/ lmontano/perelman_tratado_argumentacion.pdf.

Ramos, M. G., Sánchez, W., \& Huapaya, E. (2014). Argumentación lógica como herramienta para la formación ciudadana en estudiantes de 3ro de secundaria: Una propuesta didáctica. Lima. Perú: Comité Latinoamericano de Matemática Educativa A. C.

Romero, A. (2012). Del curriculum por objetivos al curriculum por competencias.

Sanchez, M. (2015). De la Retórica a la Teoría de la Argumentación, Cap I en Argumentación: Teoría $Y \quad$ Práctica. Disponible En: Https:/Www.Academia.Edu/9868933/Argumentaci\%C3\%93n_Teor\%C3\%8da_Y_Pr\%C3\% 81ctica. 